\title{
Compared Efficacy of Intravesical Bcg Vs Mitomycin-C, and Other Dual Therapies in Non-Muscle Invasive Bladder Cancer
}

\author{
Verdeja-Robles $\mathrm{CA}^{1,2 *}$, Turcio-Aceves $0^{1,3}$, Hernandez-Ibarra $\mathrm{MA}^{1,2}$, Barragan-De la Cruz $\mathrm{M}^{1,2}$, Sanchez-Pereda \\ $\mathrm{D}^{3}$, Ordaz-Contreras $\mathrm{A}^{3}$ and Martínez-Gómez $\mathrm{AY}^{3}$
}

${ }^{1}$ Spanish Hospital of the Spanish Beneficiencia Society

${ }^{2}$ Popular Autonomous University of the State of Puebla

${ }^{3}$ Autonomous University of Guadalajara

Submission: May 10, 2018; Published: May 15, 2018

"Correspondence Address: Verdeja-Robles CA, Hospital Español de la Sociedad de Beneficiencia Española, Universidad Popular Autonoma del Estado de Puebla, Mexico, Email: cesaralonso.verdeja@upaep.edu.mx

\begin{abstract}
Introduction: The aim of this study is to compare the efficacy of treatment with intravesical immunotherapy using the Calmette-GuérinBacillus against other intravesical chemotherapeutics in the non-muscle invasive bladder cancer, letting us elucidate the benefits of its therapeutic application, as well as the benefits of apply a dual therapy with BCG and other chemotherapeutics.

Materials and methods: A systematic research and review was assesed in 2018 of articles referring to treatment with the Calmette-Guérin Bacillus in non-muscle invasive bladder cancer, using the following sources: Clinical Key, Pub Med, Up to date and Proquest.

Results: The treatment of non-muscle invasive bladder cancer with high-risk (CIS and T1) continues to be a challenge, being actually the induction and maintenance intravesical BCG after transurethral resection the most effective therapy in reducing remittances compared to intravesical chemotherapeutic agents, however if BCG is unsuccessful, radical cystectomy may be preferred, although in cases where it's contraindicated or also the patient may be denied, combinations with interferon 2ab, mitomycin c, gemcitabine, use of thermotherapy and EMDA (electromotive drug administration) may be used to increase the expectancy of life in patients with no other alternatives.

Conclusion: Intravesical BCG immunotherapy has resulted the first-line and more effective treatment to reduce recurrences of nonmuscle invasive bladder tumors, nevertheless, if contraindications or lack of adherence to the treatment, it can be modified to intravesical chemotherapeutics, although they are associated with higher relapses at short and long term.
\end{abstract}

Keywords: Bacillus of calmette-guérin; Non-muscle invasive bladder cancer; Intravesical quemotherapeutics; Side effects; Interferon $\alpha 2 \beta$.

\section{Introduction}

More than 100 years ago there was an idea of the association of a mycobacterial disease with a decrease in the incidence of cancer. In 1926, Centanni and Rezzesi were the first to document the treatment with Bacillus of Calmette-Guérin (BCG) against cancer in mice. This rose the curiosity of being able to modulate the immune response against cancer by using a microorganism, so Holmgren started using BCG as a vaccine against cancer in 1935 [1]. Due to the success of increased remission in experimental studies in mice in 1960, it was also used for melanoma, leukemia, colon cancer and lung cancer; however, the results had variable successes, which led to more studies. There was an association but causality was not demostrated. It was up to the moment that Mathe and collaborators showed promising results by publishing that $1 / 3$ of the patients with lymphoblastic leukemia treated with BCG remained in remission compared with those who received placebo, who died without remission data; being Morales and collaborators in 1976 the first to use Bacillus Calmette-Guérin in bladder cancer [1,2].

Although the results were promising, the advance of chemotherapy resulted in the abandonment of BCG with the exception of its use in bladder cancer, which until the year 2016 has been documented that the application of intravesical immunotherapy with BCG was superior to intravesical chemotherapy as an adjuvant to the resection of the noninvasive tumor, thus decreasing the percentage of recurrences after surgical procedure [3].

Currently, bladder cancer is a major health problem, representing the most common malignancy of the urinary tract, being the $6^{\text {th }}$ most common cancer in men and the $19^{\text {th }}$ in women, leading to percentages of $7 \%$ of cancer cases in men and $2 \%$ in women with an average age of 63 years at the time of diagnosis, leading to recurrences at $80 \%$, and progression to invasive 
muscle tumor at 5 years in $45 \%$ of the cases, with tobacco being the triggering factor in more than $50 \%$ of cases [3-9]. In recent years, intravesical BCG monotherapy has been compared with other intravesical agents such as mitomycin c, gemcitabine, epirubicin, interferon alfa, which has been shown that intravesical immunotherapy with BCG post-transurethral resection of nonmuscle invasive bladder cancer, offers lower rates of recurrence in the case of bladder cancer Ta, T1, CIS, so when there is an advance of cancer affecting muscle, the treatment changes and in most cases the use of radical cystectomy is considered. The gold standard when there is extension to muscle is radical cystectomy, thus allowing to reduce the risk of tumor progression and death $[1,3-8,10,11]$.

For the effectivity of the treatment, it's necessary an interaction between Calmette-Guèrin Bacillus and tumoral cells, thus this treatment is reserved for patients with non-muscle invasive bladder cancer. The mechanism of action is based on the patient's immune response, so a poor immune response leads to a decrease response to the treatment, however, an exaggerated immune response will generate adverse effects, so it's important to know the contraindications of its use such as: immunosuppression, gross hematuria, history of sepsis due to BCG, active infection of the urinary tract and traumatic cauterization [1].

The main action of BCG in the bladder is to mediate the production of adhesion and co stimulators molecules after it's internalization into the tumor cells, thus increasing the binding capacity of $\mathrm{T}$ lymphocytes and neutrophils to receptors, leading to an enhanced activity against tumor cells resulting in their apoptosis and inhibition of their replication $[1,7,12]$.

When performing the intravesical BCG instillation, the role played by adhesion to the urothelium is crucial, which is mediated by a glycoprotein known as fibronectin binding protein expressed on the surface, which confers the ability to internalize into the tumor cells, inducing in the first instance the release of chemotactic cytokines, with an increase in macrophages, lymphocytes and natural cytotoxic cells, functioning as a cycle in which the tumor cell is inducing the activity of the antigenpresenting cells, leading to a major recognition of them, inhibiting their proliferation and finally death of the tumor cells $[1,2,12,13]$.

Now a days there is no mechanism known for sure, however, there is clear evidence of increased cytokines in the urine and plasma, of people receiving BCG immunotherapy against patients who don't receive it; so it has been seen that IL-1, IL6, IL-8, IL-10 and ICAM1 are present in the first week postinstillation, IFN gamma at 3 weeks post-instillation, IL-2 and TNF -alpha at 4 weeks post-instillation, being relevant since the cytokines that are first expressed directly and indirectly produce activation of the $\mathrm{T}$ lymphocytes with the subsequent production of tumor necrosis factor and interferon, which makes us understand that BCG treatment needs a minimum of 4 weeks to show its effectiveness due to the production and expression of modulating substances $[2,11,13]$. At the time of diagnosis between $70-80 \%$ of bladder cancers are non-muscle invasive, leading to an increase in the research and knowledge about the best therapeutic options, being transurethral bladder tumor resection the first-line of treatment, however it has been shown that when is combined with BCG maintenance therapy, increases the effectivity and remission rates, demonstrating superiority to the use of chemotherapy and induction BCG $[1,7,8,14,15]$.

\section{Objectives}

i. To compare effectiveness of intravesical treatment with mitomycin C Vs Bacillus Calmette-Guérin (BCG) for non-muscle invasive bladder cancer.

ii. To compare the effectiveness of intravesical treatment with Bacillus Calmette-Guérin (BCG) + Interferon $\alpha 2 \beta$ for non-muscle invasive bladder cancer.

\section{Materials and Methods}

(Figure 1).

\section{Results}

\section{Mitomycin-c vs bcg}

Currently, intravesical immunotherapy with BCG offers the best rates of reduction of recurrences; however, some authors compare the effectiveness of BCG with mitomycin-C (MMC). The mitomycin-C has shown similar results than the BCG however, in the maintenance periods the BCG has better results than the CMM [5,14-16].

Mondal and collaborators compare the use of mitomycin-c $40 \mathrm{mg}$ dissolved in $50 \mathrm{ml}$ of saline for two hours, for 6 weeks vs. BCG $120 \mathrm{mg}$ in $50 \mathrm{ml}$ of saline for two hours. For six continuous weeks; during a six-month follow-up period, a lower recurrence rate was found in the BCG group compared to the MMC [17]. Regarding the adverse effects, the group treated with BCG presented a higher percentage (70\%), compared to the group treated with mitomycin c (40\%); Among those that were cystitis, fever, hematuria and urinary retention, cystitis being the most frequently encountered complication, however, the adverse effects of mitomycin- $\mathrm{C}$ when they occur are more severe [14,17,18-21].

It's considered failure to treatment with BCG in the following situation:

i. When an invasive muscle tumor is detected during treatment.

\section{ii. Tumor refractory to BCG:}

iii. When a non-invasive high-grade muscle tumor (G3) is detected at 3 months post-treatment.

iv. Presence of CIS at 3 and 6 months. (Follow up at 6 months after treatment is important because up to $50 \%$ of patients with history of CIS of 3 months of evolution present a complete response to treatment until this period of time) [8] (Table 1). 


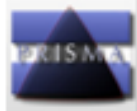

PRISMA FLOW DIAGRAM
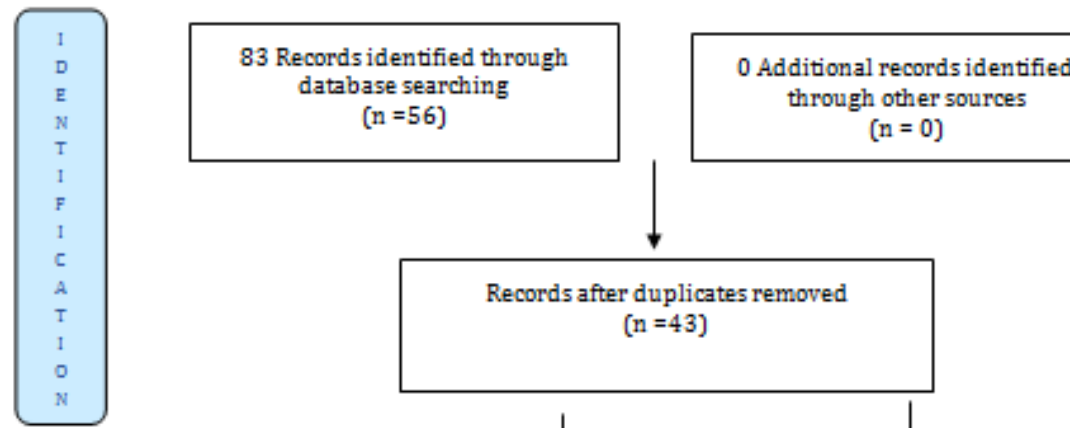

Additional records identified through other sources $(n=0)$
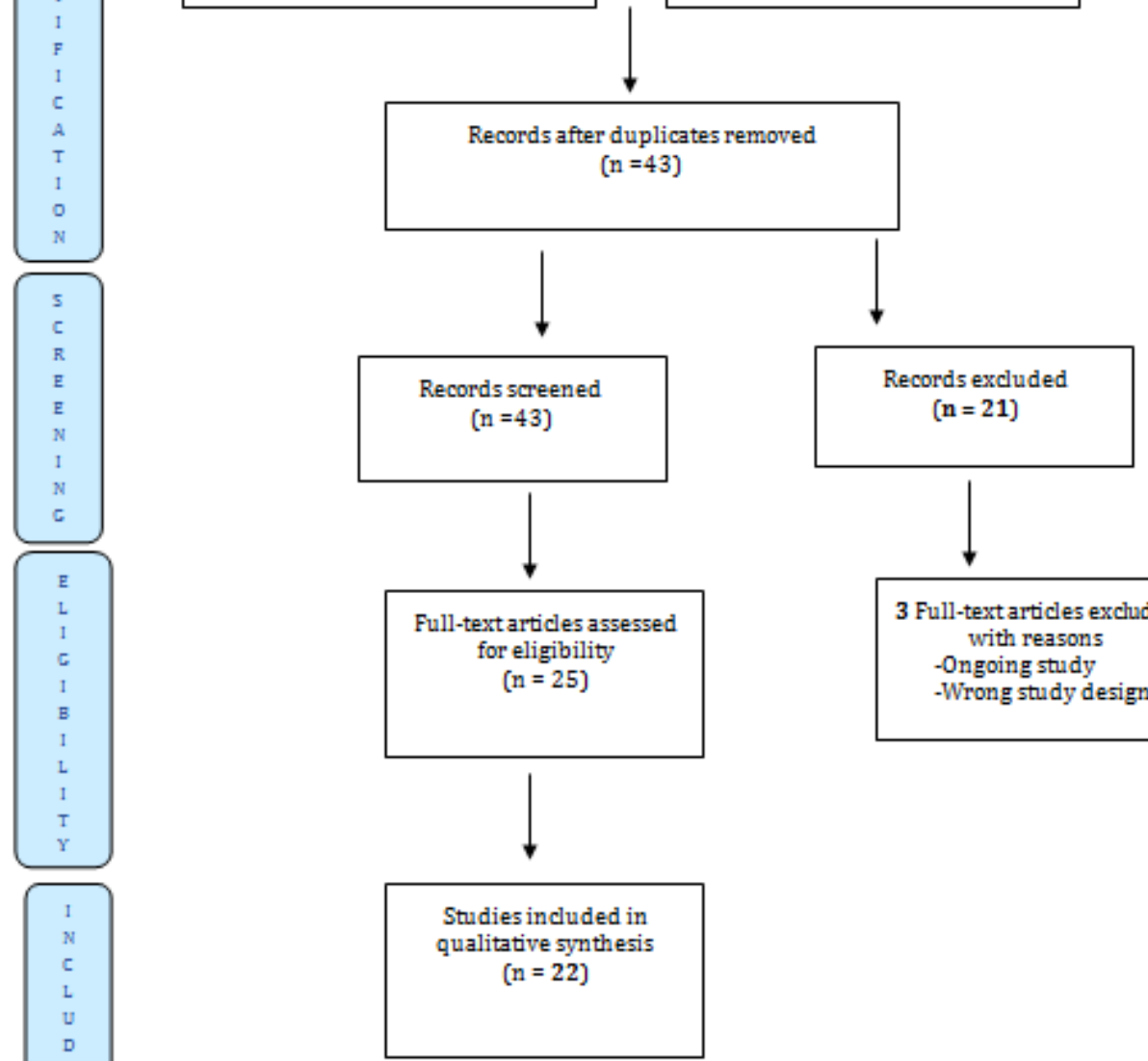

3 Full-text articles excluded, with reasons - Ongoing study -Wrong study design
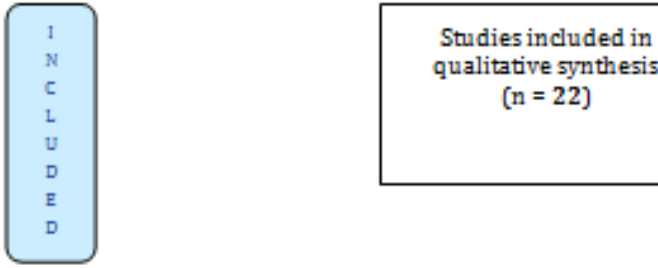

ludedin $(n=22)$

Figure 1: A systematic search was carried out in the year 2018 of articles referring to the treatment with Bacillus Calmette-Guérin in noninvasive muscle bladder cancer, using the following sources: Clinical Key, Pub Med, Up to Date and Proquest.

Table 1: Compared Side Effects BCG vs. Mitomycin C.

\begin{tabular}{|c|c|c|c|c|c|c|c|c|}
\hline \multicolumn{4}{|c|}{ Hematuria } & \multicolumn{2}{c|}{ Dysuria } & \multicolumn{2}{c|}{ Fever } & \multicolumn{2}{c|}{ Cystitis } \\
\hline Study group & Mitomycin C & BCG & Mitomycin C & BCG & Mitomycin C & BCG & Mitomycin C & BCG \\
\hline Mondal et al. [16] & $0 \%$ & $0 \%$ & - & - & $0 \%$ & $28.57 \%$ & $42.10 \%$ & $57.14 \%$ \\
\hline Friedrich et al. [18] & $9.40 \%$ & $11.60 \%$ & $20.50 \%$ & $17.30 \%$ & $2.40 \%$ & $9.30 \%$ & - & - \\
\hline DI Stasi et al. [20] & $16.70 \%$ & $72.70 \%$ & - & - & $0 \%$ & $19.40 \%$ & $25 \%$ & $66.70 \%$ \\
\hline Krege et al. [21] & $3 \%$ & $6 \%$ & - & - & $0 \%$ & $18 \%$ & $16 \%$ & $34 \%$ \\
\hline
\end{tabular}

\section{Bcg + Interferon A2 $\beta$ As Dual Therapy}

More than $40 \%$ of patients do not respond to BCG immunotherapy, the combination with interferon- $\alpha 2 \beta$ (INF$\alpha 2 \beta$ ) has been proposed, aiming to decrease the progression of cancer in patients and the frequency of the cystectomies $[5,7,11]$. Interferon- $\alpha 2 \beta$ is an immunomodulatory molecule, with anti-

proliferative activity, increasing the action of modulating the immune system. Patients with non-invasive muscle bladder cancer show improvement with intravesical BCG treatment, which ranges from $55 \%$ to $65 \%$, however, $30 \%$ to $40 \%$ of patients present with cancer recurrence, so in the last Five-year evaluations were made of the adjuvant treatment of BCG with interferon $\alpha 2 \beta$ (INF- $\alpha 2 \beta)[7,14]$. 


\section{Cancer Therapy \& Oncology International Journal}

Table 2: Follow-up and recurrence free survival rates in patients treated with BCG + IFN- $\alpha 2 \beta$ therapy.

\begin{tabular}{|c|c|c|c|c|}
\hline First Author, Year & Treatment modality. & Number of Patients & Recurrence-free Survival & Progression \% \\
\hline Correa et al. [7] & BCG + IFN- $\alpha 2 \beta$ & 44 & $\begin{array}{c}38.6 \% \text { and 27\% at } 12 \text { and 24 months } \\
\text { respectively }\end{array}$ & - \\
\hline Joudi et al. [22] & BCG + IFN- $\alpha 2 \beta$ & 1007 & $\begin{array}{c}59 \% \text { (BCG naïve), 45\% (BCG failure), at 24 } \\
\text { months }\end{array}$ & - \\
\hline O'Donnel et al. [23] & BCG + IFN- $\alpha 2 \beta$ & 40 & $63 \%$ and 53\% at 12 and 24 months respectively & $12 \%$ \\
\hline
\end{tabular}

In the studies it was found that patients to whom intravesical BCG caused resistance saw a considerable improvement of $50 \%$ by adding interferon $\alpha 2 \beta$, which were evaluated at 12 and 24 months, resulting in a cancer recurrence-free rate. $63 \%$ and $53 \%$ respectively $[7,19]$, it should be noted that many times patients who do not demonstrate a significant improvement with BCG in the first 6 months hardly respond to therapy in conjunction with INF- $\alpha 2 \beta$, what in these patients leads to the use of radical cystectomy, which generates multiple complications such as hernias, pyelonephritis, ureteroenteric fistulas [14] (Table 2).

\section{Intravesical Chemotherapy + Hyperthermia}

It was demonstrated by the Synergo Europe that in the case of failure of the treatment with BCG there are other options with chemotherapy, which can be combined with the hyperthermia method, since it increases the intravesical absorption of certain chemotherapeutic agents, among them one of the most used is mitomycin c $[3,14]$. This process is performed by certain artifacts that are capable of applying local intravesical heat and allow the use of the chemotherapeutic at the same time, this is achieved by placing a 20 French urethral catheter, by which the chemotherapeutic is applied to a radio frequency of $950 \mathrm{MHz}$; Among the most known artifacts in this procedure is the ALBA hyperthermia system and the BSD-2000 system [3].

Efficacy was recognized when results were obtained from 51 patients who failed BCG treatment and at the start of thermotherapy + mitomycin C, a complete initial response of $92 \%$ was obtained and remained in $50 \%$ of the patients at 2 years, therefore, it is essential to verify the need to change the treatment in patients who fail BCG therapy, since once it crosses the basement membrane and invades muscle, the treatment is modified and mortality increases $[3,11,14]$.

\section{EMDA-MMC + BCG}

The combination of mitomycin c administered (which tries to equal intravesical concentrations as the concentrations of

Table 3: Percentage of disease free rate at follow up by BCG / EMDA the bladder wall) has an effectiveness close to that generated by treatment with BCG $[5,7,14]$. This treatment has proven to be effective, since it applies an electric current through the plasma membrane of the urothelial cells, allowing faster introduction of the drug, obtaining higher concentrations in a shorter time of the applied intravesical agent [5,14]. In 2003 the efficacy of administering BCG vs. EMDA-MMC (electromotive drug administration-mitomycine c) treated with a six-week induction course was compared, having complete response rates to cancer of $52.8 \%$ and $55.5 \%$ for EMDA-MMC and BCG, respectively [2022]. They also showed high-risk non-muscle invasive bladder cancer recurrence rates at 43 months of $52.8 \%$ for both EMDAMMC and BCG, this being the reason why researchers decided to include BCG to the EMDA-MMC scheme with the intention of decreasing recurrence rates and improving disease-free rates [5].

By applying BCG before intravesical chemotherapy, an immune response is established that leads to infiltration of cytokines and T lymphocytes, which increases the permeability of the tissue to the absorption of MMC, greatly increasing absorption by the use of EMDA in comparison with passive diffusion, however, costs are higher and tolerance remains a challenge $[5,14]$. The sequential BCG scheme with EMDA-MMC is based on the intravesical application of these compounds for 9 weeks, with BCG being applied once in the first and second week by means of Foley catheter at doses of $81 \mathrm{mg}$ dissolved in $50 \mathrm{ml}$ of saline water for a period of time. 2 hours and the EMDA application by electrodes consecutively to the MMC instillations at a dose of $40 \mathrm{mg}$ dissolved in $100 \mathrm{ml}$ of saline water once a week, this process being repeated until completing the 9 weeks [5]. After a follow-up period of 88 months, after treatment of BCG / EMDA-MMC vs. BCG, a disease-free period of 69 months vs. 21 months of BCG was demonstrated, recurrence of $41.9 \%$ and $57.9 \%$ for BCG / EMDA- MMC and BCG respectively and progression to muscle invasive tumor of $9.3 \%$ for BCG / EMDAMMC versus $21.9 \%$ of BCG [5,14] (Table 3).

MMC treatment.

\begin{tabular}{|c|c|c|c|}
\hline Treatment with BCG / EMDA-MMC & Number of Patients & Percentage & Recurred or Progressed No. Patients, (Percentage) \\
\hline 1-year disease-free rate & 86 & $86 \%$ & $12 / 86(14 \%)$ \\
\hline 2-year disease-free rate & 71 & $93 \%$ & $5 / 71(7 \%)$ \\
\hline
\end{tabular}

Gan C, Amery S, Chaterton K, Khan MS, Thomas K, O’Brien T. risk non muscle invasive bladder cancer (HR-NMIBC) - two year Sequential BCG / Electromotive drug administration Mitomycin $\mathrm{C}$ (EMDA-MMC) as the standard intravesical regimen in high 


\section{Cancer Therapy \& Oncology International Journal}

\section{Conclusion}

Bladder cancer currently continues to occupy the first place in incidence of tumors that affect the urinary tract representing a therapeutic challenge, so it's essential to accept that treatment with intravesical immunotherapy with BCG has been found to be the most effective in reducing recurrences of non-recurring tumors. invasive muscle in the bladder; However, if there are contraindications or lack of adherence to treatment, other alternatives can be used, among which are the intravesical chemotherapeutics, with MMC being the most effective but with more severe adverse effects, so the goal in the treatment of Non invasive muscle bladder cancer is a combination of transurethral resection and maintenance BCG instillations, which has proven to be the most effective in the short and long term.

\section{References}

1. Alexandroff AB, Nicholson S, Patel PM, Jackson AM (2010) Recent advances in bacillus Calmette-Guerin immunotherapy in bladder cancer. Immunotherapy 2(4): 551-560.

2. Sidi GR, Glickman MS, Bochner BH (2014) The mechanism of action of BCG therapy for bladder cancer-a current perspective. Nature Reviews Urology 11: 153-162.

3. Veeratterapillay R, Heer R, Johnson MI, Persad R, Bach C (2016) HighRisk Non-Muscle-Invasive Bladder Cancer-Therapy Optiones During Intravesical BCG Shortage. Curr Urol Rep 17(9): 68.

4. Mcaninch JW, Lue TF (2014) Smith y Tanagho Urología General. $18^{\text {th }}$ (edn), University of California School of Medicine, San Francisco California, USA, pp. 310-322.

5. Gan C, Amery S, Chaterton K, Khan MS, Thomas K, et al. (2016) Sequential BCG / Electromotive drug administration Mitomycin C (EMDA-MMC) as the standard intravesical regimen in high risk non muscle invasive bladder cancer (HR-NMIBC) - two year outcomes. The Journal of Urology 195(6): 1697-1703.

6. Babjuk M, Böhle A, Burger M, Capoun O, Cohen D, et al. (2017) EAU Guidelines on Non-Muscle-invasive Urothelial Carcinoma of the Bladder: Update 2016. European Urology 71(3): 447-461.

7. Andres F Correa, Katherine Theisen, Matthew Ferroni, Jodi K Maranchie, Ronald Hrebinko, et al. (2015) The Role of Interferon in the Management of BCG Refractory Nonmuscle Invasive Bladder Cancer. Advances in Urology 656918: 1-6.

8. Gontero P, Bohle A, Malmstrom P, O'Donnell M, Oderda M, et al. (2010) The Role of Bacillus Calmette-Guérin in the Treatment of Non-MuscleInvasive Bladder Cancer. European Urology 57(3): 410-429.

9. Jung J, Gudeloglu A, Kiziloz H, Kuntz G, Miller A, et al. (2017) Intravesical electromotive drug administration for non-muscle invasive bladder cancer. Cochrane Database of Systematic Reviews.

10. Cockerill PA, Knoedler JJ, Frank I, Tarrell R, Karnes RJ (2015) Intravesical gemcitabine in combination with mitomycin as salvage treatment in recurrent non-muscle-invasive bladder cancer. BJU
International 117(3): 456-462.

11. Steinberg RL, Thomas LJ, Mott SL, O’Donnell MA (2016) Bacillus of Calmette Guérin (BCG) Treatment Features with Non-Muscle Invasive Bladder Cancer: A Data-Driven Definition for BCG and Unresponsive Disease. Bladder Cancer 2(2): 215-224.

12. Liu X, Dowell AC, Patel P, Viney RP, Foster MC, et al. (2014) Cytokines as effectors and predictors of responses in the treatment of bladder cancer by bacillus Calmette-Guérin. Future Medicine 10(8): 14431456.

13. Kassouf W, Black P (2017) Treatment of primary non-muscle invasive urothelial bladder cancer. Wolters Kluwer 6-18.

14. Redorta JP, Solsona E, Angulo J, Fernández JM, Madero R, et al. (2016) Estudio Retrospectivo De Diferentes Opciones de Tratamiento Conservador Con Bacilo de Calmette Guerin en el Carcinoma urotelial Vesical T1G3: Terapia de Mantenimiento. ACTAS Urológicas Españolas 40: 370-377.

15. Fernandez-Gomez J, Solsona E, Unda M, Martinez-Piñeiro L, Gonzalez M, et al. (2018) Prognostic Factors in Patients with Non-MuscleInvasive Bladder Cancer Treated with Bacillus Calmette Guerin: Multivariate Analysis of Data from Four Randomized CUETO Trials. Eur Urol 53(5): 992: 1001.

16. Mondal HP, Yirang K, Mukhopadhyay C, Adhikary SS, Dutta B, et al (2016) Prospective Randomized Study between Intravesical BCG and Mitomycin-C for Non-Muscle-Invasive Urothelial Carcinoma of Urinary Bladder Post TURBT. Bangladesh Journal of Medical Science 15(1): 7478.

17. DeBruyne FM, van der Meijden AP, Geboers AD, Franssen MP, van Leeuwen MJ, et al. (1998) Mitomycin C intravesical therapy in superficial bladder cancers. Urology 31(3 Suppl): 20-25.

18. Joudi F, Smith B, O'Donnell M (2006) Final results from a national multicenter phase II trial of combination bacillus Calmette-Guérin plus interferon $\alpha-2 B$ for reducing recurrence of superficial bladder cancer. Urol Oncol 24(4): 344-348.

19. Friedrich M, Pichlmeier U, Schwaibold H, Conrad S, Huland H (2007) Long-Term Intravesical Adjuvant Chemotherapy Further Reduces Recurrence Rate Compared with Short-Term Intravesical Chemotherapy and Short-Term Therapy with Bacillus Calmette-Guérin (BCG) in Patients with Non-Muscle-Invasive Bladder Carcinoma. European Urology 52(4): 1123-1130.

20. Di Stasi S, Giannantoni A, Stephen R, Capelli G, Navarra P, et al. (2003) Intravesical Electromotive Mitomycin C Versus Passive Transport Mitomycin C for High Risk Superficial Bladder Cancer: A Prospective Randomized Study. J Urol 170(3): 777-782.

21. Krege S, Giani G, Meyer R, Otto T, Rubben H (1996) A Randomized Multicenter Trial of Adjuvant Therapy in Superficial Bladder Cancer: Transurethral resection only versus transurethral resection plus mitomycin $\mathrm{C}$ versus transurethral resection plus bacillus CalmetteGuerin. The Journal of Urology 156(3): 962-966.

22. O’Donell M, Krohn J, Dewolf W (2001) Salvage Intravesical Therapy with Interferon- $\alpha 2 \mathrm{~B}$ Plus low dose Bacillus Calmette-Guerin is effective in patients with superficial Bladder Cancer in whom Bacillus CalmetteGuerin Alone Previously Failed. J Urol 166(4): 1300-1305. 
This work is licensed under Creative Commons Attribution 4.0 License

DOI: 10.19080/CTOIJ.2018.10.555798
Your next submission with Juniper Publishers will reach you the below assets

- Quality Editorial service

- Swift Peer Review

- Reprints availability

- E-prints Service

- Manuscript Podcast for convenient understanding

- Global attainment for your research

- Manuscript accessibility in different formats ( Pdf, E-pub, Full Text, Audio)

- Unceasing customer service

Track the below URL for one-step submission https://juniperpublishers.com/online-submission.php 